\title{
A comparative approach to cue competition with one and two strong predictors
}

\author{
IRINA BAETU, A. G. BAKER, and CHRISTINE DARREDEAU \\ McGill University, Montréal Québec, Canada \\ and \\ ROBIN A. MURPHY \\ University College, London, England
}

\begin{abstract}
The relative validity effect (Wagner, Logan, Haberlandt, \& Price, 1968) demonstrated that a strong cue or cause reduces responding to, or judgments of, a weaker cue or cause. We report two experiments with human subjects using relative validity preparations in which we investigate one- and twocue competition effects. Previously, we investigated the effect using instrumental and Pavlovian conditioning preparations with rats. In the first experiment, we used a procedure analogous to the animal preparations. In the second experiment, we used a different probabilistic procedure. The results with humans and rats are very similar. In each species we find similar interference with processing the moderate predictor with one or with two strong competitors. These results are not well predicted by most associative models.
\end{abstract}

Attempts to understand complex information processing with associative models have a long history in modern psychology. Psychological associationists, such as Hull (1943) and Spence (1940), assumed that associative bonds between stimuli and responses were strengthened through reinforcement. Moreover, they made the assumption that associations between two events were formed more or less independently of any other associations that were formed at the same time. In spite of the simplicity of this theory, which postulates gradual, continuous formation of associations, they were able to provide a sophisticated analysis of phenomena in discrimination learning that previously were thought to require noncontinuous learning processes. Noncontinuous processes, including hypothesis testing and selective attention, originally were invoked to account for phenomena that seemed beyond the explanatory scope of simple associative theory. These phenomena seemed to require complex explanations because rats and pigeons appeared to learn the relationship between events rather than to form specific associations between each cue and the outcome. These empirical phenomena included the easy-to-hard effect (transfer along a continuum, Lawrence, 1952) and the peak shift (Spence, 1937).

In the late 1960s, two series of experiments challenged the fundamental assumption that associations were formed independently of others being formed or exercised at the

This research was supported by a National Science and Engineering Research Council Grant to A.G.B. Correspondence should be addressed to A. G. Baker, Department of Psychology, McGill University, $1205 \mathrm{Dr}$ Penfield Ave, Montreal, QC, H3A 1B1 Canada (e-mail: andy.baker @mcgill.ca). same time. Kamin (1969) demonstrated how prior learning about one stimulus-outcome relationship interfered with learning a second stimulus-outcome relation. In his original demonstration of this effect, called blocking, Kamin used a conditioned suppression procedure with rats. Pairing one element A (a light or tone) with a shock $\left(\mathrm{A}^{+}\right)$prior to pairing a compound of that element and the target element $\mathrm{X}$ with shock $\left(\mathrm{AX}^{+}\right)$reduced conditioning to $\mathrm{X}$ compared with treatments in which only the compound trials $\left(\mathrm{AX}^{+}\right)$were trained. That is to say, prior training with A blocked conditioning to $\mathrm{X}$. This is one example of what are called selective associations because, although it was paired an equal number and proportion of times with the outcome (shock) in the same context (in compound with A) in both groups, X generated very different levels of responding. Because learning about $\mathrm{X}$ was influenced by learning about $\mathrm{A}$, blocking challenged the notion that associations are formed independently of one another.

Indeed, blocking has been one of the most thoroughly studied phenomena, both in traditional conditioning paradigms and in more recent research in human contingency learning and causal reasoning. The blocking effect has been used to support associative explanations of human learning (Arcediano, Matute, \& Miller, 1997; Chapman \& Robbins, 1990; Dickinson, Shanks, \& Evenden, 1984) and to study the neuroscience of attention (Rickert, Lorden, Dawson, Smyly, \& Callahan, 1979) and psychopathology (Jones, Gray, \& Hemsley, 1992), as well as more recently to explore the domains of causal and inferential reasoning (De Houwer \& Beckers, 2003; Lovibond, Been, Mitchell, Bouton, \& Frohardt, 2003; Waldmann \& Holyoak, 1992). This interest has been maintained in spite of the fact that the fundamental ex- 
perimental design used to demonstrate blocking has a number of procedural problems. These limit any conclusions about the source of the selective learning effect. For example, the experimental and control groups differ in their experience with the blocking stimulus $\mathrm{A}$, in the total number of outcomes (e.g., shocks), and whether training includes experience with noncompounded stimuli. Even though subsequent experiments have controlled for many of these methodological issues, although rarely in a single experiment, it still remains that most blocking experiments present experience with the blocking stimulus at a time that is temporally removed from experience with the compound. This introduces the possibility of interfering memory effects (e.g., primacy and recency) that confound any considerations of the pure informational value or validity of the stimuli. One possible reason for the popularity of the blocking design may be that the most popular associative model of selective associations, the Rescorla-Wagner model (Rescorla \& Wagner, 1972), makes clear predictions about blocking that are relatively independent of the conditioning parameters.

It is worth contrasting the methodological concerns with blocking with the second original selective association finding, the relative validity effect, that was reported by Wagner et al. (1968). In their basic experiment, two groups of animals received intermixed trials of two compound stimuli, each composed of the target stimulus X (a light) and one of two other stimuli A and $\mathrm{B}$ (two auditory stimuli). In the true-discrimination (TD) group, all occurrences of one compound were reinforced, whereas no occurrences of the other compound were reinforced $\left(\mathrm{AX}^{+}, \mathrm{BX}^{-}\right)$. In the pseudodiscrimination (PD) group, half of the trials with each compound were reinforced $\left(\mathrm{AX}^{+/-}, \mathrm{BX}^{+/-}\right)$. Notice that both groups received the same amount of experience with $\mathrm{A}, \mathrm{B}$, and $\mathrm{X}$. Moreover, in both groups, X was paired with the outcome on half of the trials. In spite of the similar history of $\mathrm{X}$ with reinforcement, $\mathrm{X}$ controlled much more responding in the PD group than in the TD group. According to Wagner et al., this occurred because, in the TD treatment, A (which was reinforced $100 \%$ of the time) was a relatively more valid predictor of the outcome than X, whereas in the PD treatment A, B, and X were each reinforced $50 \%$ of the time.

The relative validity effect has several procedural advantages over blocking. For instance, as we have said, unlike in blocking, the two treatments receive the same experience with A, B, X, and the outcome. As well, in contrast to blocking, the information processing is done simultaneously, so there is little concern about simple memory effects. Moreover, the basic procedure provides a discrete-trial conditioning analogue for the role of contextual or irrelevant stimuli in learning. The context is made up of unchanging stimuli that are thought to compete with or modulate responding controlled by traditional discrete stimuli. While rather rough-grained control of contextual stimuli is possible using manipulations of the environment, it is often interesting to have more experimental control. Clearly, because the target stimu- lus $\mathrm{X}$ is always present, it provides an analogue to the context and provides a number of advantages in terms of assessing behavior controlled by it. Elsewhere we have taken advantage of this analogy (Murphy, Baker, \& Fouquet, 2001b; see also Murphy \& Baker, 2004).

It is possible that the relative validity effect has received little analytic attention because even though it is predicted by the Rescorla-Wagner model (see Miller, Barnet \& Grahame, 1995), this prediction requires various a priori assumptions about the effectiveness of unconditioned stimuli during conditioning (see Gallistel, 1990, for a discussion of this assumption). The problem with this is that the theory can predict the effect or fail to predict the effect depending upon which values are chosen for the model. We will describe experiments that examine the predictions of the model for relative validity along with some new treatments that test a similar aspect of learning but do not require a priori assumptions. This is useful since it helps us decide whether the RescorlaWagner model is generally useful in its approach for modeling selective associations. But first we will describe the Rescorla-Wagner model and its predictions concerning relative validity.

\section{The Rescorla-Wagner Model and Relative Validity}

The Rescorla-Wagner model is an extension of an error correction model proposed by Bush and Mosteller (1955). Rather than positing that associations involving a cue are formed independently of other simultaneously present cues, the Rescorla-Wagner model argues that associations are influenced by all stimuli present on a learning trial. Thus, this model can account for both blocking and the relative validity effect. The amount of learning that accrues to a cue on a single conditioning trial is defined by the following formula:

$$
\Delta \mathrm{V}_{\text {cue }}=\alpha_{\text {cue }} \beta_{\mathrm{US}}\left(\lambda_{\mathrm{US}}-\sum \mathrm{V}_{\mathrm{all} \mathrm{cues}}\right) \text {. }
$$

According to this formula, the change in associative strength of a given cue $\left(\Delta \mathrm{V}_{\text {cue }}\right)$ is a function of the difference between the level of, or asymptote of, associative strength that the unconditioned stimulus (US) can support $\left(\lambda_{\text {US }}\right)$ and the sum of the associative strengths of all cues, including the experimental context, that are present on that trial $\left(\Sigma \mathrm{V}_{\text {all cues }}\right) . \lambda_{\text {US }}$ takes on a positive value on trials with the reinforcer present and is assumed to be zero on extinction trials when the reinforcer is not present. The linear operator $\left(\lambda_{\mathrm{US}}-\Sigma \mathrm{V}_{\text {all cues }}\right)$ produces the prototypical negatively accelerated learning curve. $\alpha_{\text {cue }}$ and $\beta_{\mathrm{US}}$ are learning rate parameters for the conditioned stimulus (CS) and US, respectively. They affect both the rate and, as we will see shortly, the asymptote for learning. The magnitude of each of the learning rate parameters is determined by the salience and associability of the appropriate stimulus. Generally, the ordinal predictions of the model for various treatments are independent of the learning rate parameters, so they are often ignored. However, Rescorla and Wagner (1972) pointed out that this was not the case with the relative validity 
effect since the model predicted the empirical result only if the learning rate parameter for the US was constrained.

For the Rescorla-Wagner model to account for relative validity, the learning rate parameter for the US on a reinforced trial ( $\left.\beta_{\mathrm{US}}\right)$ must be set greater than on a nonreinforced trial $\left(\beta_{\text {no US }}\right)$. This makes some sense since the presence of a reinforcer, such as shock, should be more salient than its absence. When $\beta_{\mathrm{US}}=\beta_{\text {no US }}$, the associative strength of $\mathrm{X}$ is equal in the two groups. When $\beta_{\mathrm{US}}<\beta_{\text {no US }}, \mathrm{X}$ in the TD treatment will control more responding than in the PD group. Figure 1 shows a simulation, from the Rescorla-Wagner model, of the asymptotic level of associative strength controlled by $\mathrm{X}$ for situations in which the difference between $\beta_{\mathrm{US}}$ and $\beta_{\text {no US }}$ ranges from -.9 to +.9 . This set of simulations illustrates the dependence of the Rescorla-Wagner model on the relationship between $\beta_{\mathrm{US}}$ and $\beta_{\text {no US. It shows the re- }}$ versal of relative validity that we described, and it also shows that this reversal is entirely a function of changes in associative strength of $\mathrm{X}$ in the control group (PD group). That is to say, the associative strength of $\mathrm{X}$, following a TD treatment, is independent of the difference between learning rate parameters for reinforcement and nonreinforcement, whereas it is not following PD. Murphy, Baker, and Fouquet (2001a) have described how changing the relative proportion of reinforced and nonreinforced trials has a similar influence on the relative validity effect.

\section{Two-Cue Relative Validity}

One might think that it would be easy to test the predictions of the model against the behavior of animals by simply varying the difference between the learning rate parameters for reinforcement and nonreinforcement and then observing changes in responding to $\mathrm{X}$. However, it is difficult to imagine a manipulation of the US that would make its presence less effective than its absence. The alternative that we describe here is to modify the design of the experiment. It turns out that there is a rela- tively simple manipulation of the basic relative validity design for which the Rescorla-Wagner model generates clear predictions that are independent of the relative size of the learning rate parameters for reinforcement and nonreinforcement.

In the relative validity effect, one relatively strong predictor $\left(\mathrm{A}\right.$ in $\left.\mathrm{AX}^{+} \mathrm{BX}^{-}\right)$reduces judgments of a moderate predictor $(\mathrm{X})$. What if there are two strong predictors? One can attenuate the effectiveness of a blocking stimulus (here A) by including a second cue that is also always followed by the US. Murphy et al. (2001a) made a simple modification to the basic relative validity design that allowed them to compare the effects of one and two strong predictors. This modification also generated clear, and somewhat counterintuitive, predictions from the Rescorla-Wagner model. They added a fourth cue (C) to the experiment and presented the animals with three rather than two compounds (AX, BX, and CX). In order to balance experience with the US, they used twice as many exposures to $\mathrm{CX}$ as to the other two compounds (AX, BX, and 2CX). There were two TD treatments. All $\mathrm{CX}$ trials were reinforced and no AX or BX trials were reinforced in the one-cue TD treatment (TD-1: $\mathrm{AX}^{-}$, $\left.\mathrm{BX}^{-}, 2 \mathrm{CX}^{+}\right)$. In this treatment, $\mathrm{C}$ was a single strong predictor. In the two-cue TD treatment (TD-2), all AX and $\mathrm{BX}$ trials were reinforced but no $\mathrm{CX}$ trials were $\left(\mathrm{AX}^{+}, \mathrm{BX}^{+}, 2 \mathrm{CX}^{-}\right)$. Here $\mathrm{A}$ and $\mathrm{B}$ represent two strong predictors. Finally, in the PD treatment, half of each type of trial was reinforced $\left(\mathrm{AX}^{+/-}, \mathrm{BX}^{+/-}, 2 \mathrm{CX}^{+/-}\right)$.

As in the traditional relative validity design, the target cue $\mathrm{X}$ is paired with the outcome on $50 \%$ of all trials in all three treatments, and, at least by this independent measure, $X$ should generate similar levels of behavior in all three groups. In the TD-1 treatment, as in the traditional relative validity experiment, the strong cue, here $\mathrm{C}$, had a higher probability of reinforcement than $\mathrm{X}$ and also signaled all of the outcomes, as did $\mathrm{X}$, whereas in the TD-2 treatment, A and B each was reinforced $100 \%$ of the time but each only predicted $50 \%$ of the outcomes.

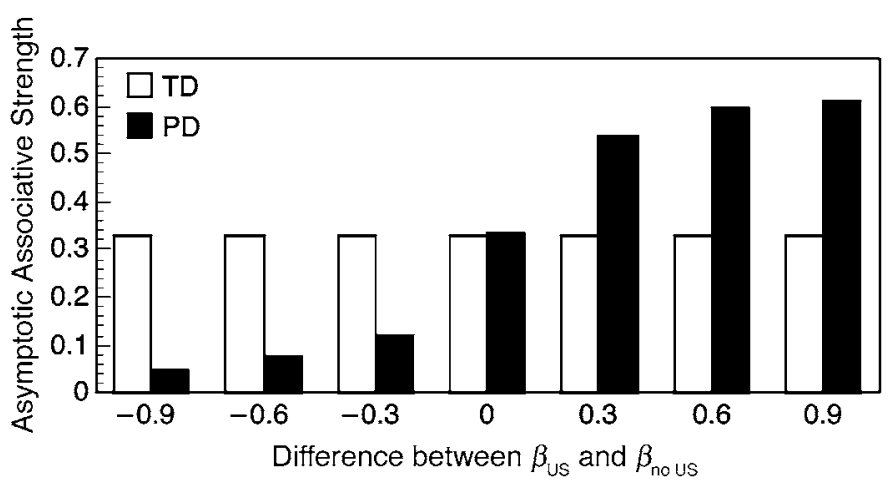

Figure 1. Asymptotic associative strengths to $X$ from simulations of the Rescorla-Wagner model for the relative validity effect for various differences between $\boldsymbol{\beta}_{\mathrm{US}}$ and $\boldsymbol{\beta}_{\text {no US }}$. For positive differences, $\boldsymbol{\beta}_{\text {no US }}$ was held constant at .1, and for negative differences, $\beta_{U S}$ was held constant at .1. 
Empirically this might reduce their ability to block $\mathrm{X}$ since, although $\mathrm{X}$ is reinforced only $50 \%$ of the time, it is the only cue that precedes $100 \%$ of the outcomes.

Interestingly, the Rescorla-Wagner model is sensitive to both the proportion of cue presentations that are followed by reinforcement and to the total proportion of outcomes that are predicted by a cue. Murphy et al. (2001a) reported simulations from the Rescorla-Wagner model for differences between the learning rate parameters for reinforcement that were similar to those we report here in Figure 1 for the standard relative validity preparation. The model predicted that TD-1 and TD-2 treatments would have markedly different effects on the associative strength controlled by X. They found that, for all differences between learning rate parameters, there was a constant difference between associative strength controlled by $\mathrm{X}$ in the TD- 1 and TD- 2 treatments. This difference was thus independent of differences in $\beta$ s. Specifically, $\mathrm{X}$ acquired less associative strength (i.e., the relative validity effect was predicted to be greater) in the TD-1 than in the TD-2 treatment. As in the traditional procedure, the associative strength controlled by $\mathrm{X}$ in the $\mathrm{PD}$ treatment was not parameter free and was a function of $\beta$ difference. As in the earlier simulation, with negative $\beta$ differences, the relative validity effect was predicted to reverse. Interestingly, the relative validity in the TD-2 treatment (i.e., the difference between TD-2 and PD) was predicted to be smaller than the difference between TD-1 and TD-2 at all differences simulated. In the rightmost panel of Figure 2, we report the largest difference we simulated and thus the case in which the difference between TD-2 and PD was greatest. Clearly the theory predicts that the difference between TD-1 and TD-2 must be at least of the same magnitude as the difference between TD-2 and PD, and in most cases it is larger.

Murphy et al. (2001a) reported two experiments using rats to test this prediction. The first experiment used a classical conditioning paradigm in which brief $(10-\mathrm{sec})$ audiovisual stimuli predicted presentations of food pellets. The conditioned response in this preparation was the tendency for animals to insert their heads into the food magazine during the presentation of a signal that predicts food. Separate groups were trained with the TD-1, TD-2, and PD treatments described above. Following training, responding to $\mathrm{X}$ was assessed in the three groups. The results of this test are reproduced in the left-hand panel of Figure 2. It is clear from this figure that there was a large and equivalent relative validity effect in both the TD- 1 and TD- 2 treatments. There was no evidence for the Rescorla-Wagner prediction that the difference between TD-1 and TD-2 would be as large as or larger than that between TD-2 and the PD control.

In a second experiment, Murphy et al. (2001a) used an instrumental preparation in which the last leverpress on variable-length trials was either reinforced or not. Again audiovisual stimuli served as the cues A, B, C, and $\mathrm{X}$. Following training on the TD-1, TD-2, or PD discriminations, responding to nonreinforced $\mathrm{X}$ presentations was assessed. As with the Pavlovian preparation, there was a strong interference effect in both the TD-1 and TD-2 treatments and no evidence for a large difference between TD-1 and TD-2. This result is shown in the middle panel of Figure 2.

\section{EXPERIMENT 1}

We have been carrying out a program of research studying analogies between the information processing in human causal reasoning and animal conditioning (Baker \& Mercier, 1989; Baker, Mercier, Vallée-Tourangeau, Frank, \& Pan, 1993; Baker, Murphy, \& Vallée-Tourangeau, 1996; Baker, Murphy, Vallée-Tourangeau, \& Mehta, 2001). We have found empirical similarities between the information processing in conditioning and in causal reasoning; but, more than that, we have found associative theories, including the Rescorla-Wagner model, to be very

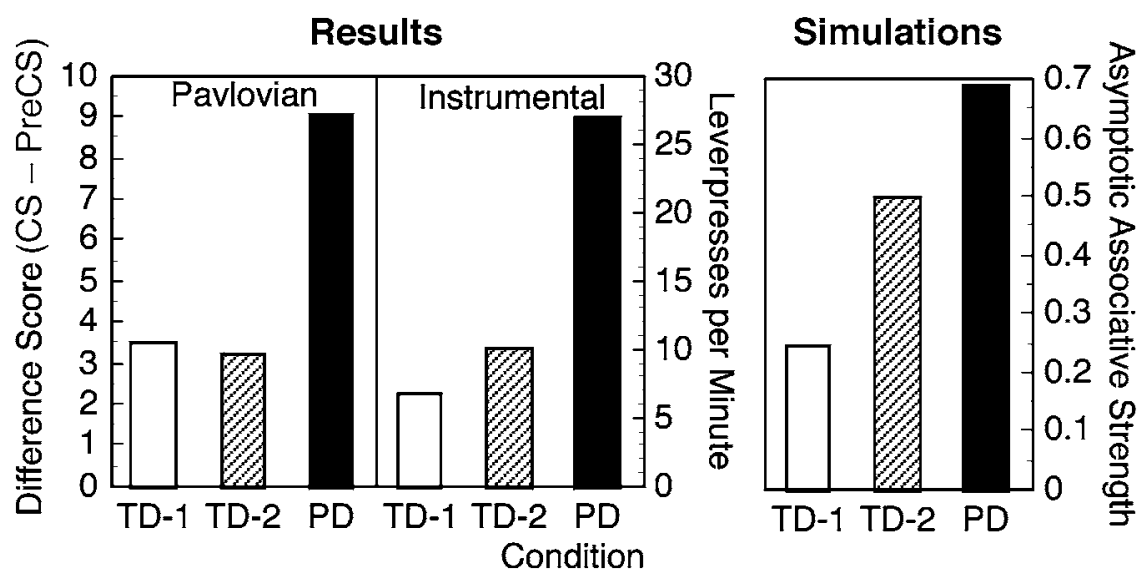

Figure 2. The left panels show results of the test to the target $X$ for the Pavlovian and the instrumental two-cue relative validity experiments using rats. The right panel shows simulations from the Rescorla-Wagner model with large $\beta$ differences $\left(\beta_{\mathrm{US}}=.9\right.$ and $\left.\beta_{\text {no US }}=.1\right)$. 
useful in understanding causal reasoning. Thus, it is of particular interest to determine whether the findings of the animal experiments we have just described extend to human causal reasoning.

The first experiment used a human version of the relative validity design (Matute, Arcediano, \& Miller, 1996; Van Hamme \& Wasserman, 1994). Human participants were told that they were to imagine being physicians whose goal was to discover which foods gave patients allergic reactions. The participants were told that they would observe the contents (the food predictors) of a number of meals that a patient had eaten. They would also be told whether or not the meals had been followed by an allergic reaction (the outcome). The foods that the meals were composed of were analogues of the cues in the animal experiments. The allergy outcome was analogous to the reward or reinforcer. Each meal consisted of a compound of two foods (e.g., shrimp and strawberries). Each patient would have meals consisting of two of three foods (A, B, X). Each patient received an equal number of $\mathrm{AX}$ and $\mathrm{BX}$ trials. So, in the TD procedure, all $\mathrm{AX}$ meals resulted in an allergic reaction but none of the $\mathrm{BX}$ meals did $\left(\mathrm{AX}^{+}, \mathrm{BX}^{-}\right)$. In the PD procedure, half of the presentations of each meal were paired with the allergy $\left(\mathrm{AX}^{+/-}, \mathrm{BX}^{+/-}\right)$. Following each condition, the participants were asked to rate how likely it was that a participant would have an allergic reaction following a meal of $\mathrm{X}$ alone. When asked this question, the participants showed a relative validity effect similar to the animal conditioning result. In spite of the fact that $X$ had been paired with the allergy on $50 \%$ of all meals in both conditions, the participants judged it to be much more likely to cause a reaction in the PD than in the TD treatment.

The goal of Experiment 1 was to determine whether two strong predictors would have the same effect on ratings of a moderate predictor as would a single perfect predictor. This experiment attempts to extend the findings of Murphy et al. (2001a) in animal learning to human contingency judgments using the same experimental design. This experiment is important because it increases the evidence of similarities between human information processing and conditioning, and further tests one of the useful associative accounts of human learning. The design compared the TD-1 treatment (i.e., $\mathrm{AX}^{-}, \mathrm{BX}^{-}, 2 \mathrm{CX}^{+}$) with the TD-2 treatment (i.e., $\mathrm{AX}^{+}, \mathrm{BX}^{+}, 2 \mathrm{CX}^{-}$) and included a (PD) control (i.e., $\mathrm{AX}^{+/-}, \mathrm{BX}^{+/-}, 2 \mathrm{CX}^{+/-}$).

\section{Method}

Participants. Twenty-four university undergraduates (21 females and 3 males) participated in this experiment for extra credit in a psychology course.

Materials. The experiment was programmed on a PC computer using E-Prime Version 1.1. Participants were tested on an individual basis.

Procedure. As in Murphy et al. (2001a), there were three conditions: true-discrimination-1 (TD-1), true-discrimination-2 (TD-2), and pseudodiscrimination (PD). Each discrimination condition involved four cues (A, B, C, and X) paired in three compounds: AX, $\mathrm{BX}$, and $\mathrm{CX}$. There were $6 \mathrm{AX}$ trials, $6 \mathrm{BX}$ trials, and $12 \mathrm{CX}$ trials in each condition, for a total of 24 trials per condition. In Condi- tion TD-1, AX and BX trials were never followed by an allergic reaction, whereas CX trials always were. In Condition TD-2, AX and $\mathrm{BX}$ trials were always followed by an allergic reaction, whereas $\mathrm{CX}$ trials never were. In Condition PD, all trial types were reinforced $50 \%$ of the time. Three sets of foods were used in Experiment 1 . A different food set was used in each condition for each participant and an equal number of participants experienced each food set in each condition. Cue X either was beef, strawberries, or bran. Cue A either was wheat, basil, or peaches. Cue B either was garlic, corn, or pork. Cue $\mathrm{C}$ either was bananas, chicken, or ketchup.

The participants completed all three conditions. The order of treatments was counterbalanced. Each condition began with instructions nearly identical to those used by Van Hamme and Wasserman (1994) except that four foods were mentioned instead of three. The instructions are shown in Appendix A.

Following instructions, the 24 trials were presented with a 1-sec intertrial interval in random order. During each trial, the participant could see the names of two of four foods with a picture of the food beside each name. The occurrence of the allergic reaction was indicated beneath the foods. On nonreinforced trials, "no allergic reaction" was written in yellow beside the picture of a healthy person, while on reinforced trials "ALLERGIC REACTION" was written in red beside the picture of the same person with a severe facial allergic reaction. Pictures of three different persons were used in the three conditions. The order of the pictures was counterbalanced across participants and conditions. At the end of each condition, participants were asked to rate each food on a scale ranging from 0 to 8 , where 0 meant that the food was definitely not the cause of the allergic reaction, 4 meant it possibly was, and 8 meant it definitely was.

\section{Results and Discussion}

Average ratings for Cues A, B, C, and X in all three conditions are plotted in the bottom panel of Figure 3 and simulations from the Rescorla-Wagner model (with a $\beta$ difference of .8) are plotted in the top panel. The cues that were always reinforced during training ( $\mathrm{A}$ and $\mathrm{B}$ in TD-2 and C in TD-1) were given almost maximal average ratings. A one-way analysis of variance (ANOVA) on the three reinforced cues confirmed that they did not differ reliably $[F(2,46)<1]$. Conversely, cues that were never reinforced (i.e., A and B in TD-1 and C in TD-2) were given average ratings close to or exactly zero. Indeed, all participants rated A in TD- 1 and C in TD-2 at zero, and 23 of 24 subjects in TD-1 rated B at zero. As seen in Figure 3, these results do not agree with the Rescorla-Wagner model, which predicts that the ratings of $\mathrm{C}$ in TD-1 should be higher than the ratings of $\mathrm{A}$ and $B$ in TD-2 and that the ratings of C in TD-2 should be lower than the ratings of $\mathrm{A}$ and $\mathrm{B}$ in TD-1. We will come back to an analysis of A, B, and C in the PD group because a direct comparison of them with $\mathrm{X}$ is more informative.

Ratings of Cue $\mathrm{X}$ were diminished in the two TD conditions relative to the PD condition. A within-subjects ANOVA on ratings of Cue $\mathrm{X}$ revealed a significant condition effect $[F(2,46)=92.3]$. Post hoc analyses indicated that this effect is mainly due to the difference between the two TD conditions and the PD condition $[F(1,23)=$ 110]. This and all other post hoc analyses used a specific error term calculated with only the difference scores involved (i.e., calculated from the means in the comparison). Bonferroni corrections for Type 1 error rejection 

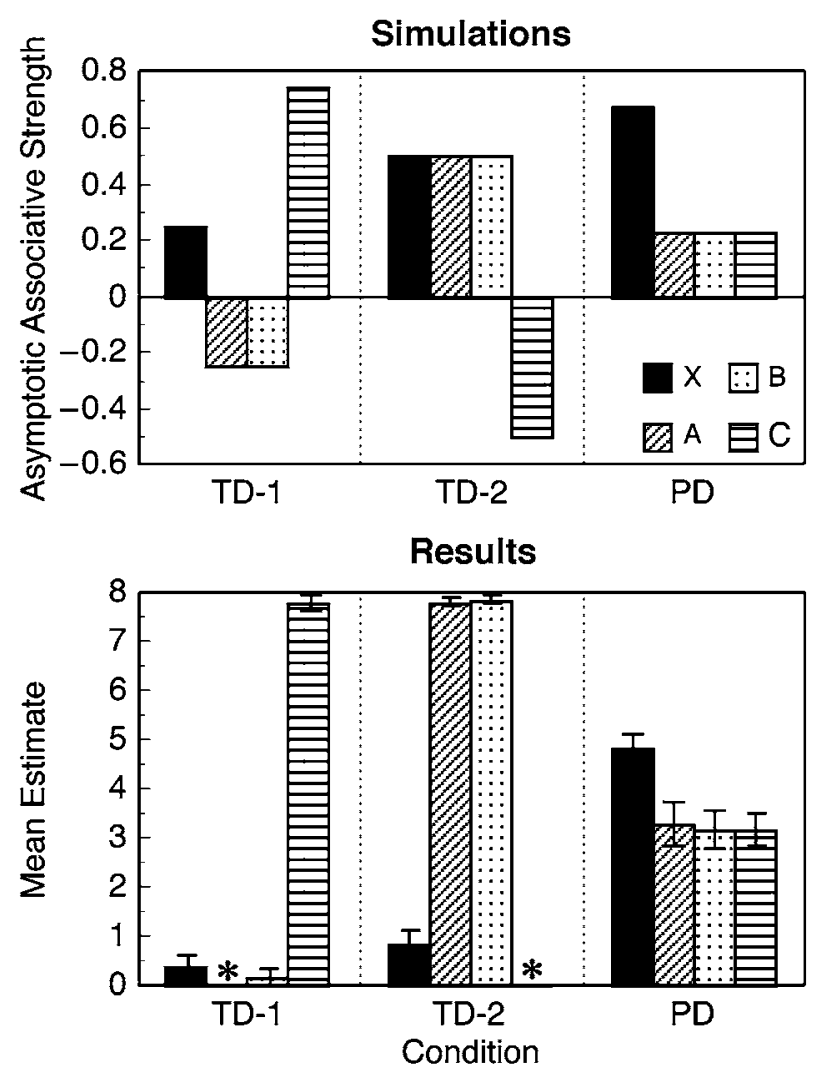

Figure 3. The top panel shows Rescorla-Wagner simulations for Experiment 1 with $\beta_{\mathrm{US}}=.9$ and $\beta_{\text {no US }}=.1$. The bottom panel shows the results of Experiment 1. The mean estimates and standard errors of Cue $A$ in TD1 and Cue $C$ in TD2 were zero (these are denoted by * in the bottom panel).

rate were used throughout. ${ }^{1}$ The difference between TD-1 and TD-2 was not reliable with the Bonferroni correction but was reliable if considered a preplanned test $[\mathrm{F}(1,23)=4.84, .05<p>.025]$. Use of a preplanned comparison may be justified here because the RescorlaWagner model does predict a difference between TD-1 and TD-2, and the design requires accepting the null hypothesis that TD-1 equals TD-2, so the Bonferroni correction might be too conservative. It should be emphasized that this reliable difference is very small and, out of 24 participants, 19 gave the same rating to $\mathrm{X}$ in the two conditions and only 5 gave a higher estimate in condition TD-2 than in condition TD-1. Moreover, the model predicts that the difference between the ratings of $\mathrm{X}$ in Conditions TD-1 and TD-2 should be greater than the difference between the ratings of $\mathrm{X}$ in Conditions $\mathrm{PD}$ and TD-2 (see the top panel of Figure 3, as well as Murphy et al., 2001a). This is not the case in Experiment 1. In fact, the difference in X estimates between TD- 1 and TD-2 is reliably smaller than the difference between TD-2 and $\operatorname{PD}[F(1,23)=39.8]$. Furthermore, for both large and small differences between $\beta_{\mathrm{US}}$ and $\beta_{\text {noUS }}$, the model predicts that Cue $\mathrm{X}$ should be able to acquire as much associative strength as the two perfect predictors, A and
$\mathrm{B}$, in TD-2. However, we found that Cue $\mathrm{X}$ was given much lower ratings than was either Cue A or Cue B in TD-2 [minimum $F(1,23)=425]$.

Finally, in Condition PD, all cues received moderate ratings, although Cue $\mathrm{X}$ was rated higher than Cues A, B, and $\mathrm{C}$, which were given roughly equal ratings. This finding is consistent with the predictions of the RescorlaWagner model in that Cue X, which was presented four times as often as A and B and twice as often as C, was able to accumulate more associative strength than A, B, or $\mathrm{C}$. Even though Cue $\mathrm{C}$ occurred twice as often as Cues $\mathrm{A}$ and $\mathrm{B}$, the Rescorla-Wagner model predicts that Cue $\mathrm{C}$ will acquire as much associative strength as $\mathrm{A}$ or $\mathrm{B}$. The ANOVA on the cues was reliable $[F(3,69)=6.46]$ and confirmed that $\mathrm{X}$ differed from each of the other cues [minimum $F(1,23)=10.7$ ], and Cues $\mathrm{A}, \mathrm{B}$, and $\mathrm{C}$ did not differ among themselves [maximum $F(1,23)=.032]$.

In conclusion, there is a great empirical similarity in the results of our two previous experiments with rats and this experiment using human participants. It seems that the information processing in these analogous preparations in very different cognitive systems is very similar. It is also clear that, although we have often reported evidence supporting the Rescorla-Wagner model as an explanation of both human causal reasoning (Baker et al., 1993; Baker et al., 2001) and animal conditioning (e.g., Murphy \& Baker, 2004), it does not do well with this new relative validity design (see also Murphy et al., 2001a). Moreover, unlike in the animal experiments, we tested judgments of A, B, and C in this experiment. We found that ratings of $\mathrm{A}, \mathrm{B}$, and $\mathrm{C}$ in the TD groups did not agree with the Rescorla-Wagner model but that ratings of the partially reinforced A, B, C, and X in the PD group did.

One common feature of this experiment and the two previous experiments using animals is that either one or two strong predictors of an outcome both reduce judgments of a moderate predictor. We will now report a second experiment that attempts to extend this finding to a different preparation that we have studied quite extensively (e.g., Baker et al., 1993), in which X is no longer a common element and in which outcomes occur in the presence and absence of $\mathrm{X}$.

\section{EXPERIMENT 2}

We have mentioned that the relative validity effect involves a comparison of the relative predictiveness of cues, and also that there are several factors that might influence a cue's absolute predictiveness. In the experiments we have discussed, X had the same absolute predictiveness in all conditions. It was paired with all of the outcomes and was followed by outcomes on half of its presentations. But this is only one value on a continuum of potential predictiveness. In these experiments, all outcomes were predicted by Cue X, but more generally in nature outcomes can be caused by a number of possible events. This requires that cues be evaluated in terms of 
how often an outcome occurs in the presence of the event, but also how often it occurs in its absence. From this perspective, a positive predictor is valid if the outcome is more likely to occur in its presence than its absence. For binary predictors and outcomes such as those we have been dealing with here, Allan (1980) has described a statistic, $\Delta p$, that provides a metric of contingency or predictiveness. $\Delta p$ is simply defined as the difference between the probability of an outcome in the presence and the absence of the predictor.

$$
\begin{aligned}
\Delta p_{\text {predictor }}= & P(\text { outcome } \mid \text { predictor }) \\
& -P(\text { outcome } \mid \text { no predictor })
\end{aligned}
$$

The experiment described next was designed to look at the effects of relative validity of cues on a moderate predictor (again X) that co-occurred with the outcome $75 \%$ of the time but, crucially, in which the outcome also occurred on $25 \%$ of occasions in which $\mathrm{X}$ was not present. Thus the presence of $X$ signaled a $50 \%$ increase in the probability of an outcome (i.e., $\Delta p_{\mathrm{X}}=.5$ ). This test is important since one possible restriction on the interpretation of the previous results could be that they reflect the effect of blocking on a cue that, like the context, is present on all trials.

Just as in the earlier experiments, there were other predictors present. The conjunctions of all experimental events, the calculations of all conditional probabilities, and the overall $\Delta p$ s are shown in Table 1. In the 1-strong condition (which is analogous to the TD- 1 treatment of Experiment 1), one predictor (A) was followed by outcomes each time it was presented and no outcomes occurred in its absence (i.e., $\Delta p_{\mathrm{A}}=1$ ). In the 2-strong (analogous to TD-2) treatment, there were two strong predictors (A and B). Each was always followed by an outcome, and together they predicted all of the outcomes, but each only predicted or was paired with half of them. Participants received exactly the same sequence of trials as in the 1-strong treatment, except that half of the A presentations were replaced with $B$ presentations. Because neither A nor B alone predicted all of the outcomes in 2strong, neither was a perfect predictor of the outcome (i.e., $\Delta p_{\text {AorB }}=.67$ ); 1 -strong has only two stimuli (A and $\mathrm{X})$ and 2-strong has three stimuli (A, B, and X). Because of this there were two controls in which $\mathrm{X}$ was presented with weak predictors (analogous to the PD control), both of which still had X as a moderate predictor (i.e., $\Delta p_{\mathrm{X}}=$ .5). In Treatment 1-weak, the second Cue A was present and it was uncorrelated with the outcome. In Treatment 2weak, there were two alternative cues present (A and B) and each of these was uncorrelated with the outcome. The probability of the outcome in the presence and absence of the uncorrelated stimuli was .5 (i.e., $\Delta p_{\mathrm{A} \text { or B }}=0$ ).

Again we derived predictions from the RescorlaWagner model. Unlike the predictions for the TD-1 and TD-2 treatments of Experiment 1, these predictions for the 1-strong and 2-strong treatments are not parameter free, but for reasonable sets they are very similar. The top panel of Figure 4 shows simulations for $\mathrm{X}$ (again
Table 1

Conjunctions of All Experimental Events, Calculations of All Conditional Probabilities, and Overall $\Delta p$ s for Experiment 2

\begin{tabular}{clcccc}
\hline Row & Trial Type & 1-Weak & 1-Strong & 2-Weak & 2-Strong \\
\hline 1 & AX & $6 / 8$ & $12 / 12$ & $3 / 4$ & $6 / 6$ \\
2 & $\mathrm{X}$ & $6 / 8$ & $0 / 4$ & $6 / 8$ & $0 / 4$ \\
3 & $\mathrm{~A}$ & $2 / 8$ & $4 / 4$ & $1 / 4$ & $2 / 2$ \\
4 & No cues & $2 / 8$ & $0 / 12$ & $2 / 8$ & $0 / 12$ \\
5 & $\mathrm{BX}$ & $0 / 0$ & $0 / 0$ & $3 / 4$ & $6 / 6$ \\
6 & $\mathrm{~B}$ & $0 / 0$ & $0 / 0$ & $1 / 4$ & $2 / 2$
\end{tabular}

Cumulative Frequencies and Probabilities for Individual Cues

\begin{tabular}{rlcccc}
7 & All X & $12 / 16$ & $12 / 16$ & $12 / 16$ & $12 / 16$ \\
8 & All no X & $4 / 16$ & $4 / 16$ & $4 / 16$ & $4 / 16$ \\
9 & $\Delta p_{\mathrm{X}}$ & .5 & .5 & .5 & .5 \\
10 & $\Delta p_{\mathrm{X} \mid \sim \mathrm{A} \sim \mathrm{B}}$ & .5 & 0 & .5 & 0 \\
11 & All A & $8 / 16$ & $16 / 16$ & $4 / 8$ & $8 / 8$ \\
12 & All no A & $8 / 16$ & $0 / 16$ & $12 / 24$ & $8 / 24$ \\
13 & $\Delta p_{\mathrm{A}}$ & 0 & 1 & 0 & .67 \\
14 & All B & - & - & $4 / 8$ & $8 / 8$ \\
15 & All no B & - & - & $12 / 24$ & $8 / 24$ \\
16 & $\Delta p_{\mathrm{B}}$ & - & - & 0 & .67 \\
\hline
\end{tabular}

Note-The top six rows show the proportion of occurrences of each event conjunction on which the sales criterion is met (e.g., row 1 shows the proportion of occurrences of the conjunction of $\mathrm{A}$ and $\mathrm{X}$ that meet the sales criterion for each treatment). X represents sports, A represents crime, and $\mathrm{B}$ represents business. The ratios represent the conditional probability of a sale given that event conjunction. The lower section shows similar ratios for each event independent of the presence or absence of the other events as well as the overall contingency for each event $\left(\Delta p_{\text {Event }}=P(\right.$ Sales $\mid$ All Event $)-P($ Sales $\mid$ All no Event $)$. For example, in row 9 for the 1 -weak condition, $\Delta p_{\mathrm{X}}=\mathrm{P}($ Sales $\mid$ All X) $\mathrm{P}($ Sales $\mid$ All no $\mathrm{X})=12 / 16-4 / 16=.5$. The conditional contrast for $\mathrm{X}$ based on the absence of $\mathrm{A}$ and $\mathrm{B}\left(\Delta p_{\mathrm{X} \mid \sim \mathrm{A} \sim \mathrm{B}}\right)$ used by probabilistic contrast theory is shown in row 10 .

with a $\beta$ difference of .8). These simulations indicate that, while there is still a small advantage for 1-strong over 2-strong, the size of the relative validity effect in both treatments is predicted to be very similar and very much larger than the difference between them. Another feature of the model concerns the prediction for the associative strength of A and B in the strong predictor treatments. These stimuli were followed by outcomes $100 \%$ of the time in both treatments, but there were outcomes in the absence of each stimulus in the 2-strong treatment, thereby reducing the actual contingency of each. The Rescorla-Wagner model predicts that there should be sensitivity to this difference. Associative strength to $\mathrm{A}$ in 1-strong is predicted to be greater than that to $\mathrm{A}$ and $\mathrm{B}$ in 2-strong.

\section{Method}

Participants and Apparatus. Twenty-four university students ( 9 males and 15 females) served as participants for extra course credit.

Procedure and scenario. The participants were told that they were consultants monitoring the sales of newspapers in four different cities. The instructions are shown in Appendix B. They were told that they were to determine whether the presence of certain categories of headlines (the predictors or cues) in the newspapers would help them meet a daily sales quota (the outcome). Sports was always the target cue X, crime was Cue A, and business was Cue B. The participants were told that they would study each newspaper for 32 days (the 32 trials). Each day they were told what categories 

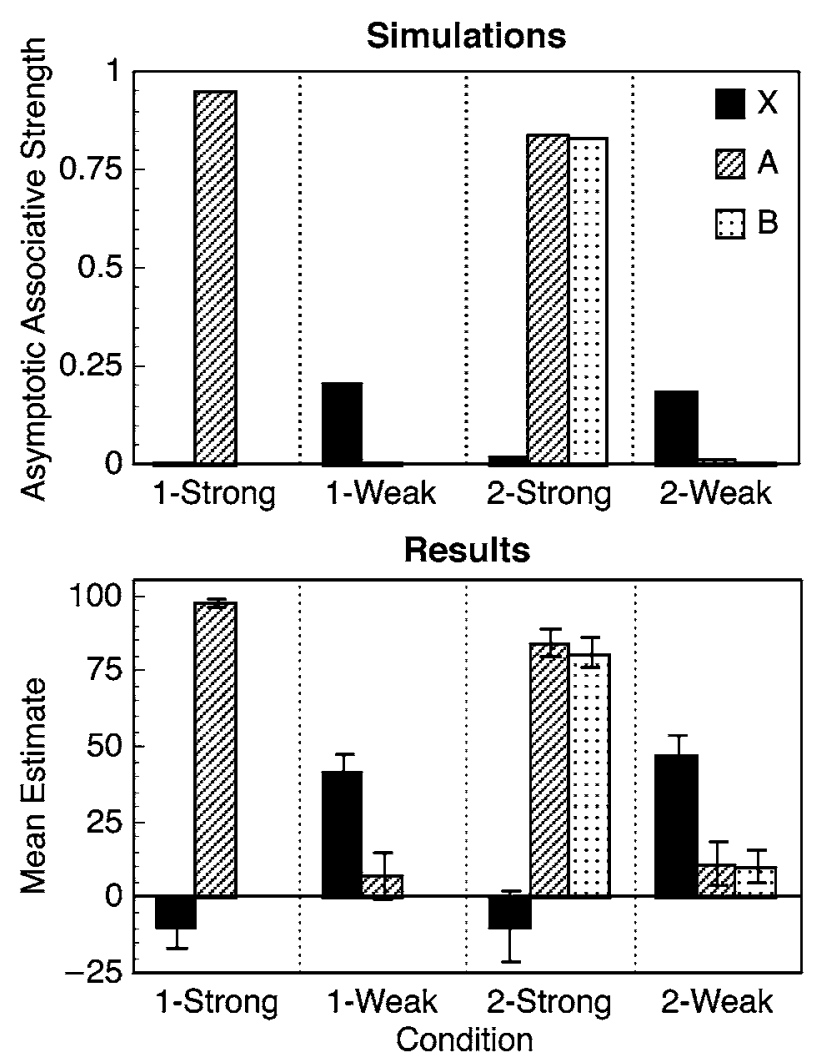

Figure 4. Rescorla-Wagner simulations for Experiment 2 with $\beta_{\mathrm{US}}=.9$ and $\beta_{\mathrm{no}} \mathrm{US}=.1$ (top panel) and results of Experiment 2 (bottom panel). In Conditions 1-strong and 1-weak, participants were trained with two cues ( $\mathrm{A}$ and $\mathrm{X}$ ), whereas in Conditions 2strong and 2-weak, participants were trained with three cues (A, $B$, and $X$ ).

of headlines the newspaper was displaying. After they had guessed whether or not the newspaper had met the sales quota that day, they were informed whether it had. They could exhibit the next trial by pushing a key. After they had been exposed to 32 trials, they were asked to estimate on a scale ranging from -100 to +100 how each class of headline influenced the outcome. The scale included negative numbers because the instructions permitted the headlines to actually interfere with sales and thus be preventative causes. This was done to allow the results of the experiment to be compared with those of other experiments using inhibitory causes.

All participants received all four treatments (1-strong, 2-strong, 1 -weak, and 2-weak) in a different order. This exhausted all 24 permutations of four events. An equal number of participants received each newspaper paired with each treatment. On a given trial, there might be no, one, or a compound of two headlines. There were no three-headline compounds. In all treatments, half of all trials ended in an outcome.

\section{Results and Discussion}

The participants' estimates for the target headline (X) are shown in the bottom panel of Figure 4. These results are very similar to those of Experiment 1 and of our two animal experiments in that there was a large and very similar relative validity effect in both the 1- and 2-strong treatments. A 2 (one vs. two competing causes) $\times 2$ (strong vs. weak) within-subjects ANOVA on estimates of $\mathrm{X}$ confirmed that the overall relative validity effect was significant $[F(1,23)=36.1]$. No other effects were reliable $(F \mathrm{~s}<1)$. What is unique about this experiment is that, unlike the results of Experiment 1, which used a modification of the traditional relative validity effect with no outcomes in the absence of the compound cues, the results of this experiment conform closely to the Rescorla-Wagner model. Although the model does predict a small difference between the 1-strong and 2-strong effect size, this difference is quite small and is not parameter independent. In fact with some reasonable parameters it can be very small indeed. Thus it seems reasonable to assume that our experiment would not have the power to detect this small difference if it existed.

As expected, the mean estimates of A and B hovered around 10 in the weak predictor control treatments and did not differ $[F(2,46)<1]$. As mentioned above, estimates of $\mathrm{A}$ in 1-strong and $\mathrm{A}$ and $\mathrm{B}$ in 2-strong are of theoretical interest both because a difference is predicted by the Rescorla-Wagner model and because comparing them contrasts estimates based on proportion of trials with an outcome (A and B were always followed by the outcome on $100 \%$ of their occurrences) with estimates based on overall contingency $(\Delta p)$. As Figure 4 indicates, the estimates for the 1-strong treatment, in which $\Delta p_{\mathrm{A}}=1$, were very high for $\mathrm{A}$, whereas those for $\mathrm{A}$ and $\mathrm{B}$ were lower in the 2-strong treatment, in which $\Delta p_{\mathrm{A} \text { or }}$ ${ }_{\mathrm{B}}=.67$. This difference is reliable $[F(2,46)=8.19]$. Comparisons of A in 1-strong with both $\mathrm{A}$ and $\mathrm{B}$ in 2strong were both reliable [minimum $F(1,23)=9.92$ ] Estimates of A and B did not differ in Treatment 2-strong $[F(1,23)<1]$. It might be noted that the participants had received more experience with $\mathrm{A}$ in 1-strong than with either of $\mathrm{A}$ and $\mathrm{B}$ in 2-strong, so the difference might have occurred because learning had not reached asymptote for A and B in 2-strong. However, in a subsequent experiment, we doubled the amount of experience and the difference remained.

A reviewer of an earlier version of this paper suggested the possibility that judgments of $\mathrm{X}$ in this experiment were not based on the overall $\Delta p$ s or overall experience with the cues, but were based on trials on which the cues occurred by themselves. For example, the target cue $\mathrm{X}$ is never paired with the outcome when it is presented alone in both strong predictor conditions. Likewise, when no cues were present, there were no outcomes. Thus $\Delta p_{\mathrm{X}}$ in the absence of other cues is equal to 0 in the strong predictor treatments but is still equal to .5 in the weak predictor treatments. It is possible that the "relative validity effect" we find here reflects attention to this subset of the data presentations (see also White, 1998). However, estimates of A and B differed in the two strong predictor treatments, and if the present argument is true, then it should also apply here. (We argued that this reflected the difference in overall $\Delta p$ s of .67 and 1.) In both strong treatments, all presentations of $\mathrm{A}$ and $\mathrm{B}$ were followed by outcomes and no outcomes occurred 
in the absences of headlines. Thus the conditional $\Delta p$ s of $\mathrm{A}$ and $\mathrm{B}$ in the absence of other events are always 1 , yet the participants in this and other experiments in our lab judge these contingencies to differ (see Baker et al., 1993). Why would participants use a subset of X experience and not of A or B experience? Moreover, it will be recalled that the 1-strong treatment uses the same contingencies as those used in our earlier research, and in those experiments we reported data that directly contradicts this possibility (e.g., Baker et al., 1993, Experiment 5, and see p. 429 for a discussion of related possibilities).

\section{GENERAL DISCUSSION}

The two experiments using human participants that we have discussed here show a great deal of empirical similarity. That is to say, we found strong and very nearly equal cue competition with either one or two strong competing cues using both preparations. The results are also similar to those of the experiments reported earlier that used animals. This implies that the relative validity effect involves a consistent and general mode of information processing that generalizes across a wide range of evolutionarily separate cognitive systems, and, perhaps, that it is a highly functional information processing strategy. More specifically, we have shown that either one or two strong cues produce similar, although not identical, interference with behaviors controlled by a moderate cue, and that the strong predictors do not need to individually predict all of the outcomes. However, these experiments do not determine all the factors that influence the relative validity or predictiveness of the strong cue. For example, the strong predictor or predictors were followed by an outcome on $100 \%$ of their presentations, and either singly, or in concert, predicted all of the outcomes. We have chosen to restrict ourselves in this paper to these situations because they generate clear predictions from the Rescorla-Wagner model. However, it is of interest to ask whether cues that do not predict all outcomes or that are followed by outcomes on a high proportion, but not all of, their presentations generate a reduction in responding to a moderate cue. In general, they seem to. Van Hamme and Wasserman $(1993,1994)$ carried out other treatments we have not described here and found that a partially reinforced predictor will reduce responding to a weaker predictor. Moreover, we have also reported data in a preparation similar to that in Experiment 2 that revealed a probabilistic relative validity effect in which the strong predictor neither always preceded an outcome nor predicted all of the outcomes (Baker et al., 2001).

We have thoroughly discussed predictions of the Rescorla-Wagner model, which generated an accurate description of the results of our Experiment 2 but did not do well on Experiment 1 or the two animal experiments. However, it is possible that other models will make accurate predictions of both results.

\section{Pearce's Generalization Model}

We have generated simulations from Pearce's (1987) generalization model of conditioning and have found that it also does not deal well with the two-cue relative validity treatment (Murphy et al., 2001a). According to Pearce, associations are not formed between individual stimulus elements and the outcome. Rather, they are formed between the total configuration of stimuli that are present on a trial. In Experiment 1, this would be Compounds AX, BX, and CX. When tested on an element, in our case the target $X$, the strength of the response is determined by the total associative strength that is generalized to X from the three compounds. Simulations of the three perfectly reinforced compounds (AX and BX in TD-2 and CX in TD-1) reach an asymptote at a value approximately equal to 1 ( 1 is the associative limit an individual compound can reach). The nonreinforced compounds reach an asymptote close to zero. In the PD treatment, all three partially reinforced compounds reach a value of approximately .67. Because each compound is composed of $\mathrm{X}$ and one other element (A, B, or C), the similarity between each compound and $\mathrm{X}$ is .5. Because of this, .5 of the associative strength of each compound generalizes to X. In the TD-1 treatment, there is only one source of strong excitation to generalize to $X(C X)$, so the strength of $X$ is predicted to be weaker than in TD-2, where there are two strong sources of generalized excitation (AX and BX). Hence judgments of $X$ in the TD-1 treatment will be very much weaker (i.e., there will be a larger relative validity effect) than in the TD-2 treatment. In the PD treatment, there are three sources of generalization of moderate excitation, compared with two strong sources in TD-2, so these two treatments predict similar strengths of responding to $\mathrm{X}$. Thus, unlike the Rescorla-Wagner model, which predicts a moderate interference effect, Pearce's generalization model predicts that there should be little or no interference in the two-cue relative validity treatment.

\section{Probabilistic Contrast Model}

There is an entire class of models that are called normative or statistical models (e.g., Cheng \& Novick, 1992). These models argue that participants remember the sequence of events they have been exposed to and then, at the time of decision, calculate some measure of contingency. This is often $\Delta p$ or a conditional $\Delta p$. A conditional $\Delta p$ is a $\Delta p$ calculated conditional on the presence or absence of one of the other cues. These theories explain selective association effects like blocking and relative validity in one of two general ways. They can argue that these experiments do not provide enough information about $\mathrm{X}$ to calculate a contingency. So participants cannot make a clear prediction unless they make assumptions about the missing information. This lack of information might reflect the fact that they have never seen X out of compound or have never seen a situation where $\mathrm{X}$ is not present. Alternatively, the theories argue 
that people are sensitive to the information that a cue provides a participant about the outcome. In the case of selective associations, the target $\mathrm{X}$ does not provide any useful information above and beyond that given by $\mathrm{A}$ and $\mathrm{B}$ (i.e., $\Delta p_{\mathrm{x}}$ conditional on the absence or presence of $\mathrm{A}$ or B is low), so it is discounted. For Experiment 2, the conditional contrasts for $\mathrm{X}$ based on the absence of $\mathrm{A}$ and $\mathrm{B}\left(\Delta p_{\mathrm{X} \mid \sim \mathrm{A} \sim \mathrm{B}}\right)$ are shown in Table 1 and it can be seen that they do predict a relative validity effect for both the 1 - and 2-strong-cue relative validity treatments. The case for Experiment 1 is not as straightforward because $\mathrm{X}$ is present on all trials, so $P($ Outcome $\mid$ no $\mathrm{X})$ is undefined. It is possible to get around this issue by considering the time between trials as no X trials (see Baker et al., 1996; Baker et al., 2001; Murphy, Vallée-Tourangeau, Msetfi, \& Baker, 2005).

\section{Comparator Models}

The discovery of selective associations led researchers to reject the notion that animals formed independent associations about cues. Blocking and the relative validity effect were argued to reflect failures to learn about one stimulus, and the Rescorla-Wagner model was developed to account for this. Although this all happened more than 30 years ago, there is still no satisfactory theoretical explanation of these phenomena using this assumption. The language was different but the old view that discriminations were solved by animals independently learning about cues and then making their decision based on the relative associative or habit strengths of various alternatives is very similar to what is now called comparator theory. Miller and his colleagues (e.g., Miller \& Matzel, 1988) have for a number of years been championing the comparator. Miller's theory is quite complicated and it is still not clear whether it deals with the basic relative validity effect (cf. Blaisdell \& Miller, 2001, p. 55; Cole, Barnet, \& Miller, 1995, p. 297). However, in principle, theories that compare or contrast associative strengths should be able to predict the relative validity effect.

We (e.g., Murphy et al., 2001a) have proposed a very preliminary related theory we call the computational comparator. According to the computational comparator, decisions about cues or causes are made by comparing the independently learned associative strength of a cue with the strength of comparator stimuli. If strong comparators have a great deal of associative strength relative to the target, then the target's ability to control behavior is reduced. Comparison stimuli are recruited by associations formed with the target stimulus during training. For example, in blocking and the relative validity effect, $A$ is presented with $X$ during training. On the test when $\mathrm{X}$ is presented alone, this $\mathrm{AX}$ association brings $\mathrm{A}$ to mind, and $\mathrm{B}$ too. When these stimuli are compared with $\mathrm{X}$, some discounting of $\mathrm{X}$ will occur. According to this version of the theory, associative strength to a cue is proportional to the conditional probability of an outcome in the presence of a cue. This is closely related to the tra- ditional idea of probability of reinforcement determining habit strength. Thus, the conditional probability of an outcome during the cue could be replaced by Hull or Spence's formulae for the independent acquisition of habit strength. Our theory suggests that the response strength for a cue depends on a comparison of the strength of the strongest stimulus present (S) with the target stimulus $(\mathrm{C})$ according to the following formula:

$$
\text { Strength of } \mathrm{C}=P(\mathrm{US} \mid \mathrm{C}) *[P(\mathrm{US} \mid \mathrm{C}) / P(\mathrm{US} \mid \mathrm{S})] \text {. }
$$

That is to say, the strength of the response to the target is modulated by the strength of the strongest comparator stimulus. Clearly because the conditional probability of the outcome in the presence of the strong stimuli in the TD-1 and TD-2 treatments is always one and the conditional probability of the outcome for the target is lower, we expect equivalent relative validity effects with TD-1 and TD-2 treatments. This theory does have some problems in the present experiments in that it has difficulty explaining the difference in estimates of A, B, C, and X in the PD treatment of Experiment 1 and has difficulty explaining the difference in estimates of the strength of $A$ in 1-strong versus $A$ and $B$ in the 2-strong treatments of Experiment 2.

This rather simple version of the comparator theory leaves out many important unresolved details, such as the following: How many stimuli are compared? Is there a negative comparison process (i.e., what is the role of $\mathrm{B}$ in $\left.\mathrm{AX}^{+}, \mathrm{BX}^{-}\right)$? Nevertheless, the general approach has little trouble with our general empirical finding that one or two strong cues generate a strong relative validity effect. It also rehabilitates many of the older explanations of discrimination learning and generalization learning.

\section{REFERENCES}

Allan, L. G. (1980). A note on measurement of contingency between two binary variables in judgment tasks. Bulletin of the Psychonomic Society, 15, 147-149.

Arcediano, F., Matute, H., \& Miller, R. R. (1997). Blocking of Pavlovian conditioning in humans. Learning \& Motivation, 28, 188199.

Baker, A. G., \& Mercier, P. (1989). Attention, retrospective processing and cognitive representation. In. S. B. Klein \& R. R. Mower (Eds.), Contemporary learning theory (pp. 85-116). Hillsdale, NJ: Erlbaum.

Baker, A. G., Mercier, P., Vallée-Tourangeau, F., Frank, R., \& PAN, M. (1993). Selective associations and causality judgments: Presence of a strong causal factor may reduce judgments of a weaker one. Journal of Experimental Psychology: Learning, Memory, \& Cognition, 19, 414-432.

BaKer, A. G., Murphy, R. A., \& Vallée-Tourangeau, F. (1996). Associative and normative models of causal induction: Reacting to versus understanding cause. In D. L. Medin, D. Shanks, \& K. Holyoak (Eds.), The psychology of learning and motivation: Vol. 34. Causal learning (pp. 1-45). San Diego: Academic Press.

Baker, A. G., Murphy, R. A., Vallée-Tourangeau, F., \& Mehta, R. (2001). Contingency learning and causal reasoning. In R. R. Mowrer \& S. B. Klein (Eds.), Handbook of contemporary learning theories (pp. 255-306). Mahwah, NJ: Erlbaum.

Blaisdell, A. P., \& Miller, R. R. (2001). Conditioned inhibition produced by extinction-mediated recovery from the relative stimulus validity effect: A test of acquisition and performance models of empir- 
ical retrospective revaluation. Journal of Experimental Psychology: Animal Behavior Processes, 27, 48-58.

BuSH, R. R., \& MOSTELLER, F. (1955). Stochastic models for learning. New York: Wiley.

Chapman, G., \& Robbins, S. J. (1990). Cue interaction in human contingency judgments. Memory \& Cognition, 18, 537-545.

Cheng, P. W., \& Novick, L. R. (1992). Covariation in natural induction. Psychological Review, 99, 365-382.

Cole, R. P., BARNet, R. C., \& Miller, R. R. (1995). Effect of relative stimulus validity: Learning or performance deficit? Journal of Experimental Psychology: Animal Behavior Processes, 21, 293-303.

DE HOUWER, J., \& BeCKERS, T. (2003). Secondary task difficulty modulates forward blocking in human contingency learning. Quarterly Journal of Experimental Psychology, 56B, 345-357.

DiCKinson, A., Shanks, D. R., \& Evenden, J. L. (1984). Judgment of act-outcome contingency: The role of selective attribution. Quarterly Journal of Experimental Psychology, 36A, 29-50.

GALlistel, C. R. (1990). The organization of learning. Cambridge, MA: MIT press.

Hull, C. L. (1943). Principles of behavior. New York: Appleton-CenturyCrofts.

Jones, S. H., Gray, J. A., \& Hemsley, D. R. (1992). Loss of the Kamin blocking effect in acute but not chronic schizophrenics. Biological Psychiatry, 32, 739-755.

KAMIN, L. J. (1969). Selective associations and conditioning. In W. K. Honig \& N. J. Mackintosh (Eds.), Fundamental issues in associative learning (pp. 42-64). Halifax, NS: Dalhousie University Press.

LAWRENCE, D. H. (1952). The transfer of a discrimination along a continuum. Journal of Comparative \& Physiological Psychology, 45, 511-516.

Lovibond, P. F., Been, S., Mitchell, C. J., Bouton, M. E., \& FroHARDT, R. (2003). Forward and backward blocking of causal judgment is enhanced by additivity of effect magnitude. Memory \& Cognition, 31, 133-142.

MAtute, H., Arcediano, F., \& Miller, R. R. (1996). Test question modulates cue competition between causes and between effects. Journal of Experimental Psychology: Learning, Memory, \& Cognition, 22, 182-196.

Miller, R. R., BARNet, R. C., \& GRahame, N. J. (1995). Assessment of the Rescorla-Wagner model. Psychological Bulletin, 117, 363-386.

MilleR, R. R., \& Matzel, L. D. (1988). The comparator hypothesis: A response rule for the expression of associations. In G. H. Bower (Ed.), The psychology of learning and motivation: Advances in research and theory (Vol. 22, pp. 51-92). San Diego: Academic Press.

MURPHY, R. A., \& BAKER, A. G. (2004). A role for CS-US contingency in Pavlovian conditioning. Journal of Experimental Psychology: Animal Behavior Processes, 30, 229-239.

MURPHY, R. A., BAKER, A. G., \& FoUQUeT, N. (2001a). Relative valid- ity effects with either one or two more valid cues in Pavlovian and instrumental conditioning. Journal of Experimental Psychology: Animal Behavior Processes, 27, 59-67.

MURPHY, R. A., BAKER, A. G., \& FoUQUet, N. (2001b). Relative validity of contextual and discrete cues. Journal of Experimental Psychology: Animal Behavior Processes, 27, 137-152.

Murphy, R. A., Vallée-Tourangeau, F., Msetfi, R., \& Baker, A. G. (2005). Signal-outcome contingency, contiguity and the depressive realism effect. In A. Wills (Ed.), New directions in associative learning (pp. 193-219). Hillsdale, NJ: Erlbaum.

Pearce, J. M. (1987). A model for stimulus generalization in Pavlovian conditioning. Psychological Review, 94, 61-73.

Rescorla, R. A., \& WAGNER, A. R. (1972). A theory of Pavlovian conditioning: Variations in the effectiveness of reinforcement and nonreinforcement. In A. H. Black \& W. F. Prokasy (Eds.), Classical conditioning II: Current research and theory (pp. 64-99). New York: Appleton-Century-Crofts.

Rickert, E. J., Lorden, J. F., Dawson JR., R., Smyly, E., \& Callahan, M. F. (1979). Stimulus processing and stimulus selection in rats with hippocampal lesions. Behavioral \& Neural Biology, 27, 454-465. SPENCE, K. W. (1937). The differential response to stimuli varying within a single dimension. Psychological Review, 44, 430-444.

SPENCE, K. W. (1940). Continuous versus noncontinuous interpretation of discrimination learning. Psychological Review, 47, 271-288.

Van Hamme, L. J., \& Wasserman, E. A. (1993). Cue competition in causality judgments: The role of manner of information presentation. Bulletin of the Psychonomic Society, 31, 457-460.

Van Hamme, L. J., \& Wasserman, E. A. (1994). Cue competition in causality judgments: The role of nonpresentations of compound stimulus elements. Learning \& Motivation, 25, 127-151.

Wagner, A. R., Logan, F. A., Haberlandt, K., \& Price, T. (1968). Stimulus selection in animal discrimination learning. Journal of Experimental Psychology, 76, 171-180.

WALDMANN, M. R., \& HolyOAK, K. J. (1992). Predictive and diagnostic learning within causal models: Asymmetries in cue competition. Journal of Experimental Psychology: General, 121, 222-236.

WHITE, P. A. (1998). Causal judgement: Use of different types of contingency information as confirmatory and disconfirmatory. European Journal of Cognitive Psychology, 10, 131-170.

\section{NOTE}

1. For the Bonferroni correction, we controlled the overall Type 1 rejection rate for a set of $k$ contrasts using the formula $\alpha / k$. $k$ is the number of a posteriori contrasts (here $F$ ratios with $1 d f$ in the numerator) $\alpha$ is the combined error rate chosen for that set of contrasts $(\alpha=.05$ throughout). Thus each of a set of two contrasts would be compared with $\alpha=.025$. 


\section{APPENDIX A}

Instructions for Experiment 1

Imagine that you are an allergist who is trying to determine the cause of an allergic reaction shortly after your patient eats dinner. You arrange that the patient eat particular foods at dinner over a series of evenings, and then report to you whether an allergic reaction followed.

The results of the test series will be shown to you on subsequent computer screens. There will be one screen for each day of the allergy test. Each screen will be displayed for 10 seconds and will indicate what the patient ate for dinner that day and whether there was an allergic reaction.

The patient will be eating two of the following four foods each day: [A], [B], [C], and [X]. After seeing all the test results, please indicate how likely you think it is that each of these foods would cause an allergic reaction by choosing the appropriate number using the following rating scale:

(0) definitely not, (4) possibly, (8) definitely.

Press the space bar to continue.

Note-A, B, C, and X were actual food names.

APPENDIX B

Instructions for Experiment 2

Conrad White has just bought a chain of newspapers. He believes that headlines sell papers. He has papers all over the country and each has a different population of readers. Each population might be interested in different stories. He hired an agency to find out which headlines tend to increase sales and which tend to decrease sales in different cities. He is particularly interested in looking at the impact that business, sports, and crime headlines have on sales. He suspects that the impact of these headlines on sales will vary across cities with different populations.

In order to investigate this possibility, the agency randomly selected a newspaper stand from each of four different cities. A profit objective was set. A quota was then determined, for each of the four newsstands, based on the number of papers sold to meet this objective.

On any day, a newspaper may have one or more of the headlines on it, or none. Each day, the headlines on the front page were recorded, as well as whether or not the profit objective for each stand was met.

You will be presented with the results of this study. The series of reports for each of the four different papers will be presented one after the other. For each paper, you will be told, day by day, which, if any, headlines appeared on the front page. Each day, you will be asked to enter whether you think the newsstand in that city reached its quota that day. After typing " $y$ " for yes or " $n$ " for no, you will immediately be informed of whether your prediction was right. You will then move on to the next day by hitting the space bar.

Please try to keep track of what happens when one or more of the headlines are present, but also when none of the headlines are present (as some populations may buy more papers than others, regardless of the headlines). However, please do not write down this information.

For each paper, you will be asked to rate how strongly each headline influences the number of sales. You will rate the impact of each headline on a scale of -100 to +100 . A positive rating means that the headline has a positive impact on sales. That is, the headline increases the chances that the newsstand will make its quota. A negative rating means that the headline has a negative impact on sales. That is, the newsstand is less likely to make the quota when the headline appears on the front page than when it does not. A rating of zero means that the headline does not have any impact on the number of sales.

The rating you enter should indicate how strongly positive or negative you think is the influence of the headline on the number of sales. A rating of 100 would indicate that the headline has a very strong positive influence on sales. A rating of 50 would indicate a more moderate positive influence of the headline.

Similarly, a rating of -100 would indicate a very strong negative impact, whereas a rating of -25 would indicate a weak negative impact. 